\title{
Spectral infrared analysis in thermal cameras with diffraction gratings
}

\author{
by R. Olbrycht*, B. Więcek* and M. Kałuża*
}

* Lodz Univ. of Technology, Institute of Electronics, Poland, robert.olbrycht@p.lodz.pl

\section{Abstract}

The aim of this paper is to demonstrate the capabilities of thermal camera equipped with diffraction grating for spectral infrared analysis. Such a rig elaborated at Institute of Electronics (Lodz University of Technology), is presented as well as exemplary results of its application for detection of $\mathrm{CO}_{2}$ concentration in the air.

\section{Introduction}

Conventional thermal camera is sensitive to infrared radiation in a given spectral range (e.g. $3-5 \mu \mathrm{m}$ ). Without additional accessories (such as bandpass filters) it is not possible to retrieve the information about the infrared radiation intensity distribution in this range. Such information may be valuable for example to detect various gases in atmosphere. Therefore the authors provide a description of approach that enables spectral analysis of infrared radiation with typical cooled thermal camera. This approach is based on using diffraction grating for the camera, which enables hyperspectral imaging. In this approach a vertical slit line image is diffracted horizontally, what means that the continuous spectral range may be acquired by the camera.

\section{Diffraction angle calculation}

Ruled reflective diffractive grating are the very typical and the most useful in infrared spectrometric instruments. In our application we used the gratings with 75 grooves per $1 \mathrm{~mm}$ density. It corresponds to the grating constant $\mathrm{d}=13.3 \mu \mathrm{m}-$ fig. 1 .

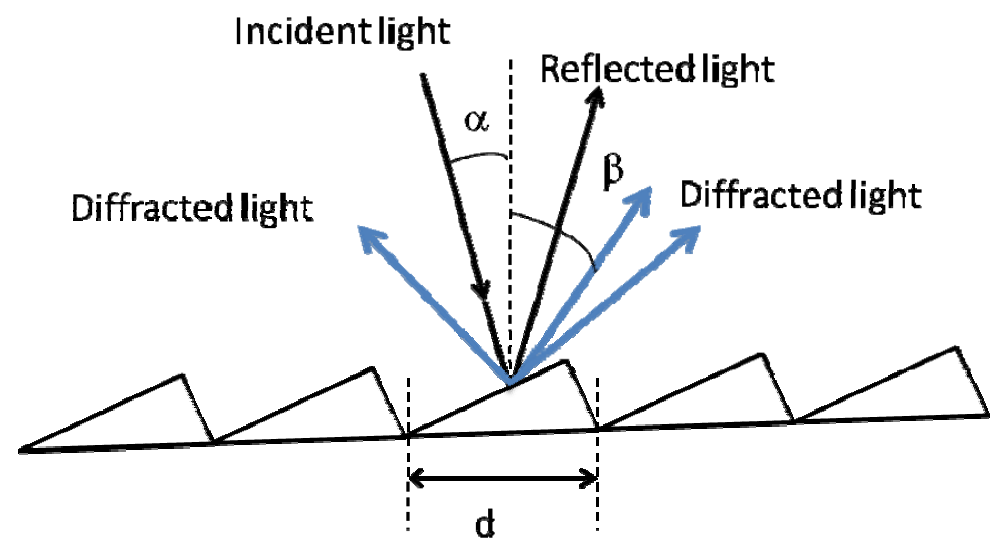

Fig. 1. Ruled reflective diffractive grating

The fundamental grating equation describes the relation between the incident $(\alpha)$ and diffractive $(\beta)$ angles together with the grating constant $d$ and the wavelength $\lambda$.

where $n$ is the diffraction order.

$$
n \lambda=d(\sin \alpha+\sin \beta)
$$

In our experiment, one used MWIR cooled camera operating in the 4-5 $\mu \mathrm{m}$ wavelength range. As it seen in fig. 2, it was possible to orient the grating to get the proper diffraction angle. 


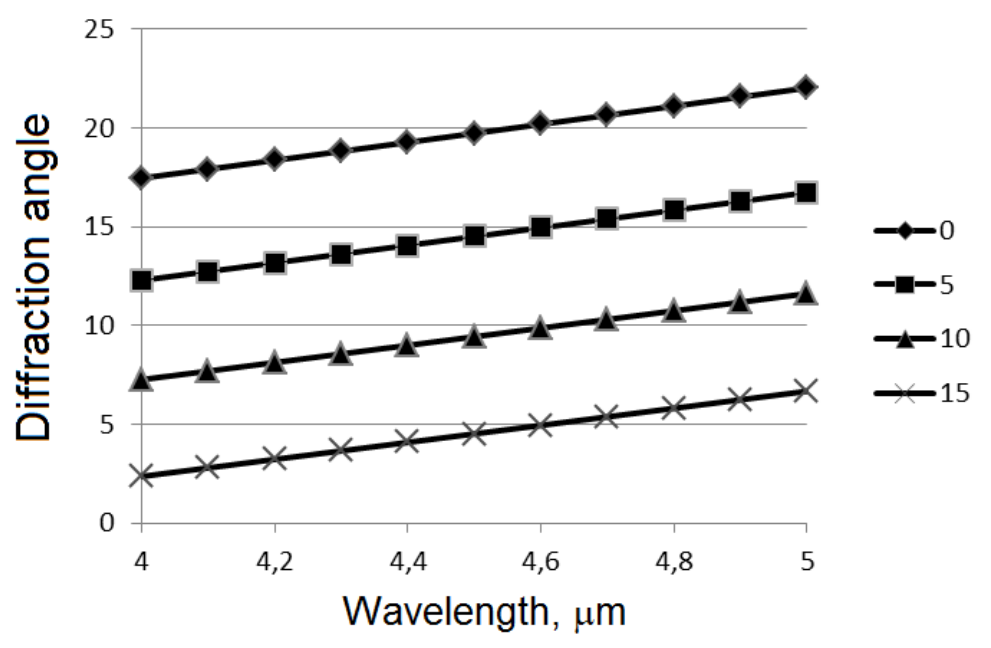

Fig. 2. Diffraction angles vs. wavelength (um) for different incident angles

\section{Description of the hyperspectral imaging rig}

The aim of the rig was to estimate the $\mathrm{CO}_{2}$ concentration in the air. The authors decided to use the simplest approach with one diffraction grating and one input slit to enable hyperspectral capability to the Cedip Titanium infrared camera. These elements were mounted externally, as shown in fig. 3 . It is possible to rotate the camera when the diffraction grating mount is fixed, but it is easier to have fixed camera and rotated grating, as in case of this experiment. Angle values were determined practically and are valid for this particular rig. Parameters of the devices involved into the rig are shown in table 1.

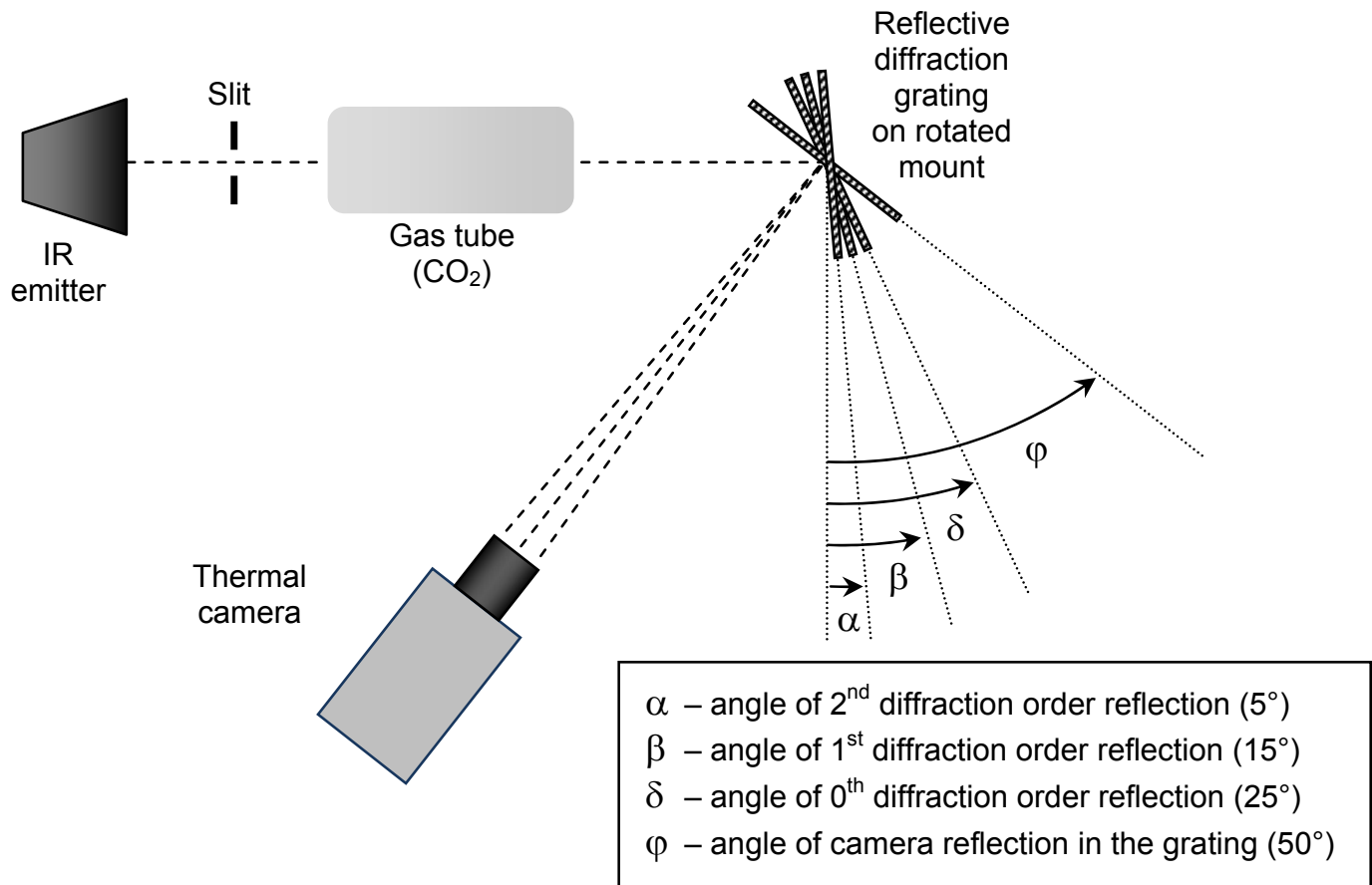

Fig. 3. The diagram of the hyperspectral imaging rig with thermal camera (diffraction angles are valid for this particular rig) 
Tab 1. Chosen parameters of the devices involved in the rig.

\begin{tabular}{|c|l|l||}
\hline \multicolumn{1}{|c|}{ Device } & Parameter & Value \\
\hline \hline \multirow{4}{*}{ IR emitter } & width & $1,5 \mathrm{~mm}$ \\
\cline { 2 - 4 } & height & $70 \mathrm{~mm}$ \\
\hline \hline \multirow{3}{*}{ Gas tube } & type & Fluke 4181 \\
\cline { 2 - 3 } & surface temperature & $170{ }^{\circ} \mathrm{C}$ \\
\cline { 2 - 3 } & surface diameter & $150 \mathrm{~mm}$ \\
\hline \hline \multirow{3}{*}{ Thermal camera } & diameter & $150 \mathrm{~mm}$ \\
\cline { 2 - 4 } & length & $500 \mathrm{~mm}$ \\
\hline \hline \multirow{3}{*}{ Diffraction grating } & model & Cedip Titanium \\
\cline { 2 - 4 } & spectral range & $3-5 \mu \mathrm{m}(\mathrm{MWIR})$ \\
\hline \hline & type & Newport 74182, ruled \\
\cline { 2 - 3 } & line density & 75 lines / mm \\
\cline { 2 - 3 } & peak efficiency & $80 \%$ \\
\cline { 2 - 3 } & blaze wavelength & $7 \mu \mathrm{m}$ \\
\hline
\end{tabular}

\section{Infrared absorption in gases}

In January 1859 Tyndall began studying the radiative properties of various gases. During his experiments he demonstrated the ability of carbon dioxide, water vapour, ozone and hydrocarbons to absorb infrared radiation. He showed also, that oxygen, hydrogen and nitrogen were almost transparent. Later investigations showed, that infrared absorption in gases is closely related to their molecule structure and the existence or lack of dipole moment.

Infrared radiation has a sufficient energy level to cause vibrations or rotations of molecules of some gases. To cause directly rotations, the molecule must have a permanent dipole moment, which means a permanent charge separation within the molecule. The requirement for infrared caused vibrations is different. To be IR-active, a particular vibration mode of gas molecule must be associated to dipole moment change. If a molecule is vibrationally active, it can also acquire and dispense rotational energy.

Diatomic molecules like $\mathrm{O}_{2}, \mathrm{H}_{2}$ or $\mathrm{N}_{2}$ have zero dipole moment and thus, those gases are almost transparent to infrared radiation.

The number of a vibrational modes in a molecule is $3 \mathrm{~N}-5$ for a linear molecules or $3 \mathrm{~N}-6$ for nonlinear molecules, where $\mathrm{N}$ is the number of atoms. Thus a $\mathrm{CO}_{2}$ molecule - the gas that was used in this study for measurements containing 3 atoms, will have $3 \times 3-5=4$ fundamental vibrational modes. The four vibrational modes of $\mathrm{CO}_{2}$ molecule are presented in fig. 4. A carbon dioxide molecule can be stretched (symmetrically $(A)$ or asymmetrically (B)) or bent in two different planes $((C)$ or $(D))$.

In the $A$ vibrational mode both carbon dioxide $\mathrm{C}=\mathrm{O}$ bonds are stretched and contracted symmetrically, whereas in the B mode they are stretched and contracted asymmetrically. The asymmetric (B) stretching is infrared active, because it is leading to dipole moment changes. This particular vibrational mode is associated with $4.26 \mu \mathrm{m}$ radiation wavelength and is the strongest $\mathrm{CO}_{2} \mathrm{IR}$ absorption band. The symmetric (A) vibration mode is not infrared active, as it is not leading to dipole moment changes. The two equal-energy bending vibrational modes in carbon dioxide ((C) and (D) in fig. 4) are identical, except that the first one is occurring in the paper plane and the second one is out of the plane. Those vibrational modes are related to infrared radiation in the $15.00 \mu \mathrm{m}$ band.

In the case of more complicated molecules, outside stretching and bending, they can vibrate in rocking and twisting modes. 


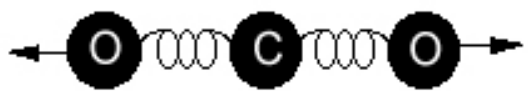

A

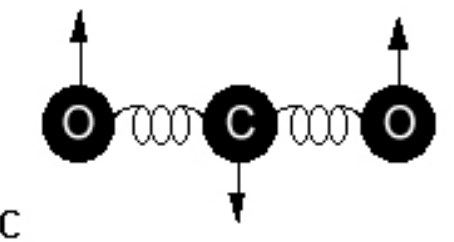

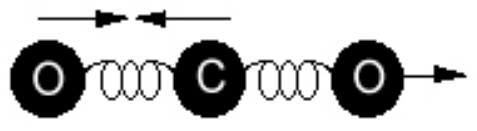

B

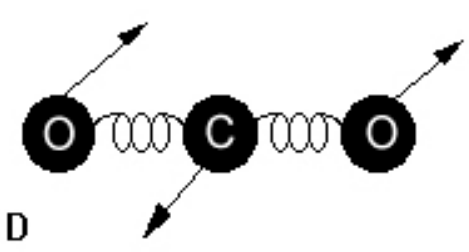

Fig. 4. $\mathrm{CO}_{2}$ molecule vibrational modes: only $B-C-D$ are leading to infrared radiation absorption.

Outside fundamental absorption bands related to molecule stretching, rotating or bending, a gas absorption spectrum may contain also overtones, combination, and difference bands. Overtones are bands that correspond to integral multiples of the fundamental absorption bands. In a molecule, two vibrational frequencies $\left(v_{1}\right.$ and $\left.v_{2}\right)$ can couple together, giving rise to a new infrared active frequency, which will be the sum (or combination) of the two interacting bands $\left(v_{\text {combination }}=v_{1}+v_{2}\right)$. In a similar way, two vibrational frequencies $\left(v_{1}\right.$ and $\left.v_{2}\right)$ can give raise to a new one, being the difference of the interacting bands ( $\left.v_{\text {difference }}=v_{1}-v_{2}\right)$. All this can lead to complicated gas infrared transmission/absorption spectra.

\section{Obtained results}

The experiment was carried out for 4 different $\mathrm{CO}_{2}$ concentrations in the gas tube. The first measurement was the reference, and the gas tube was filled with the air (under atmospheric pressure), so the $\mathrm{CO}_{2}$ concentration was at the volumetric level of $0,02-0,04 \%$ (very low). The next three measurements were repeated for low, medium and high $\mathrm{CO}_{2}$ concentrations - the gas was injected into the tube directly before the measurement. The procedure of each measurement was the same - during one second time period the camera recorded 50 frames with $1^{\text {st }}$ order reflected diffraction pattern on the grating surface. These frames were averaged in time domain to reduce noise (about 7 times in this particular case). Three differential images were calculated and shown in fig. 5:

- a) image for low $\mathrm{CO}_{2}$ concentration measurement minus image for reference measurement

- b) image medium low $\mathrm{CO}_{2}$ concentration measurement minus image for reference measurement

- c) image for high $\mathrm{CO}_{2}$ concentration measurement minus image for reference measurement

For the clearness sake, also plot d) was prepared basing on a), b) and c) spectrograms. For each $\mathrm{CO}_{2}$ concentration there is a cross-section presented, which was averaged from all rows of particular spectrogram. It enabled noticeable noise reduction and comparison between three $\mathrm{CO}_{2}$ concentrations. As expected, the higher is the concentration, the higher is the difference between the measured and reference value. Obtained plots are in agreement with known $\mathrm{CO}_{2}$ absorption spectrum (fig. 6) [2].

The proposed rig was uncalibrated, this is why the horizontal axis values are in pixels instead of wavenumbers or wavelengths. But the calibration is possible with few narrow bandpass filters, and it will be carried out in future. Even with obtained results only for $\mathrm{CO}_{2}$ it is possible to roughly calibrate the rig, what should be enough for basic spectral investigations. 
a)

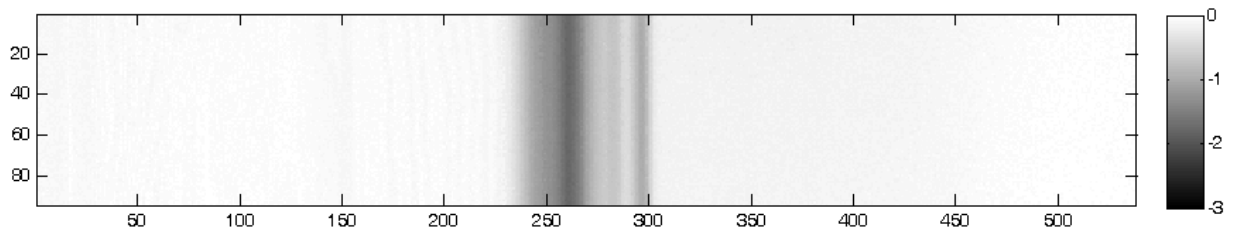

b)

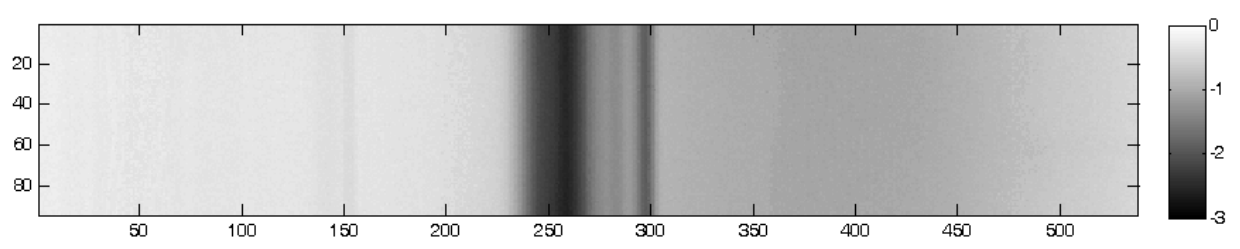

c)

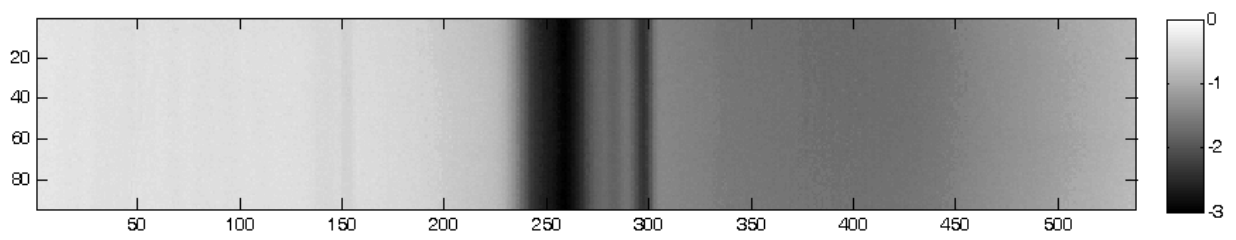

d)

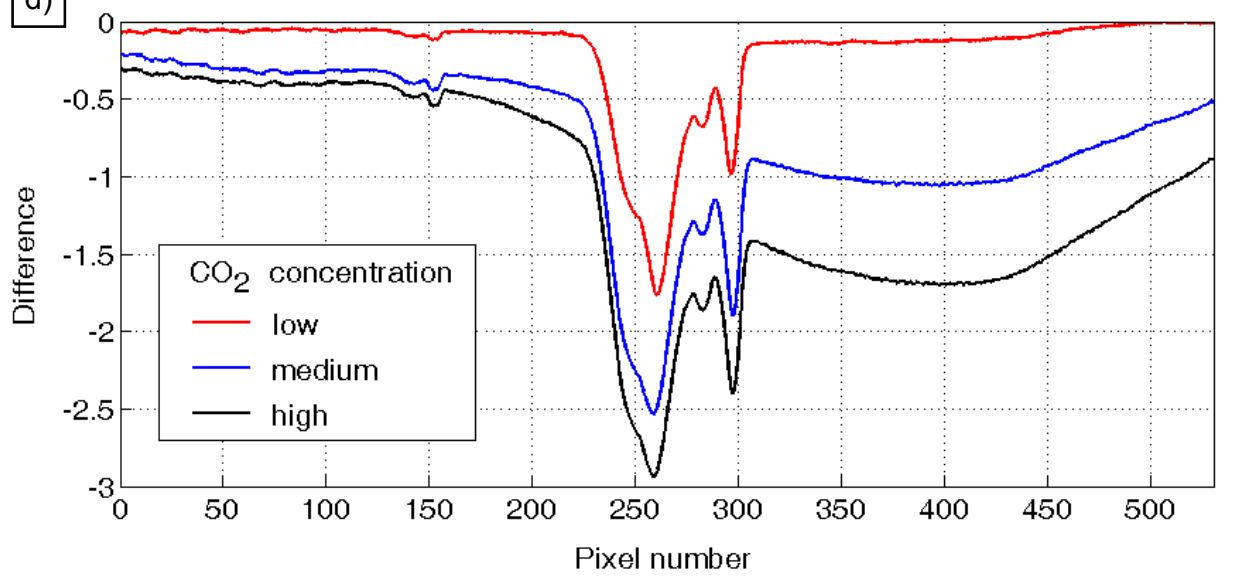

Fig. 5. The differential spectrograms for a) low, b) medium, c) high $\mathrm{CO}_{2}$ concentration in air, shown also as plots $d$ ). Vertical axes are in ${ }^{\circ} \mathrm{C}$.

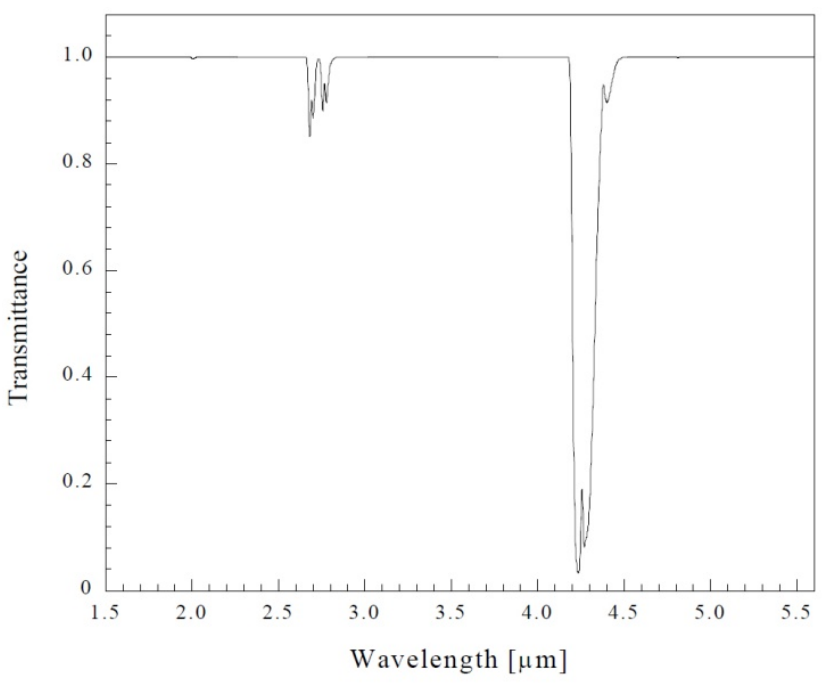

Fig. 6. Reference $\mathrm{CO}_{2}$ spectral transmittance [2]. 


\section{Conclusions}

It was demonstrated that with one diffraction grating and one slit it is possible to enable hyperspectral investigations with sensitive thermal camera (preferably a cooled one). Presented results proved that it is possible to estimate the gas content in atmosphere $\left(\mathrm{CO}_{2}\right.$ was used in this case). It is expected that the resolution of the system may be enhanced by decreasing the slit width and increasing the IR emitter temperature. Furthermore adding two mirrors to create Czerny-Turner rig may be advantageous for the purpose of hyperspectral thermal analyses. The Authors also plan to calibrate the rig with different narrow bandpass filters. Unfortunately to acquire different spectral thermograms of whole objects it is required to use push-broom scanning technique, what requires the object to be stationary. This paper is a preliminary study to this topic and further research will be carried out to improve the system and obtained results.

\section{REFERENCES}

[1] Lerner M. J., "Imaging Spectrometer Fundamentals for Researchers in the Biosciences-A Tutorial", Cytometry Part A, Special Issue: Spectral Imaging, vol. 69A, Issue 8, pp. 712-734, 2006.

[2] Denis M. Packan, Richard J. Gessman, Laurent Pierrot, Christophe O. Laux, and Charles H. Kruger, "Measurement and modeling od $\mathrm{OH}, \mathrm{NO}$, and $\mathrm{CO}_{2}$ infrared radiation in low temperature air plasma", AIAA 99-3605, American Institute of Aeronautics and Astronautics 